\title{
Turbulent Cells in Stars: Fluctuations in Kinetic Energy and Luminosity
}

\author{
W. David Arnett ${ }^{1,2}$ \\ wdarnett@gmail.com \\ Casey Meakin ${ }^{1,3}$ \\ casey.meakin@gmail.com
}

\begin{abstract}
Three-dimensional (3D) hydrodynamic simulations of shell oxygen burning (Meakin \& Arnett 2007b) exhibit bursty, recurrent fluctuations in turbulent kinetic energy. These are shown to be due to a general instability of the convective cell, requiring only a localized source of heating or cooling. Such fluctuations are shown to be suppressed in simulations of stellar evolution which use mixing-length theory (MLT).

Quantitatively similar behavior occurs in the model of a convective roll (cell) of Lorenz (1963), which is known to have a strange attractor that gives rise to chaotic fluctuations in time of velocity and, as we show, luminosity. Study of simulations suggests that the behavior of a Lorenz convective roll may resemble that of a cell in convective flow. We examine some implications of this simplest approximation, and suggest paths for improvement.

Using the Lorenz model as representative of a convective cell, a multiple-cell model of a convective layer gives total luminosity fluctuations which are suggestive of irregular variables (red giants and supergiants (Schwarzschild 1975)), and of the long secondary period feature in semiregular AGB variables (Stothers 2010; Wood. Olivier \& Kawaler 2004). This " $\tau$-mechanism" is a new source for stellar variability, which is inherently non-linear (unseen in linear stability analysis), and one closely related to intermittency in turbulence. It was already implicit in the 3D global simulations of Woodward, Porter, \& Jacobs (2003). This fluctuating behavior is seen in extended 2D simulations of CNeOSi burning shells (Arnett \& Meakin 2011b), and may cause instability which leads to eruptions in progenitors of core collapse supernovae prior to collapse.
\end{abstract}

Subject headings: stars: evolution hydrodynamics - convection -turbulence -irregular variables -Betelgeuse

\footnotetext{
${ }^{1}$ Steward Observatory, University of Arizona, 933

N. Cherry Avenue, Tucson AZ 85721

${ }^{2}$ ICRAnet, Rome, Pescara, Nice
}

\footnotetext{
${ }^{3}$ Theoretical Division, Los Alamos National Loboratory, Los Alamos, NM 875, USA
} 


\section{Introduction}

Three-dimensional fluid dynamic simulations of turbulent convection in an oxygenburning shell of a presupernova star show bursty fluctuations which are not seen in onedimensional stellar evolutionary calculations (which use various versions of mixing-length theory, MLT, Böhm-Vitense (1958)). This paper explores the underlying physics of this new phenomena.

Since the formulation of MLT Böhm-Vitense 1958), there have been a number of significant developments in the theoretical understanding of turbulent convective flow.

First, Kolmogorov (1962) and Obukhov (1962) developed the modern version of the turbulent cascade, and published in journals easily accessible in the west; the original theory (Kolmogorov 1941) was not used in MLT although it pre-dated it. This explicit expression for dissipation of turbulent velocities 1 ,

$$
\epsilon_{t u r b}=u_{r m s}^{3} / \ell_{d}
$$

where $u_{r m s}$ is the root-mean-square of the turbulent velocity and $\ell_{d}$ is the dissipation length. It is found both experimentally and numerically that $\ell_{d} \approx \ell_{C Z}$, where $\ell_{C Z}$ is the depth of the convective zone. Simulations for low-Mach number flow show that the average of this dissipation over the convective zone closely compensates for the corresponding average of the buoyant power (Arnett, Meakin, \& Young 2009). This additional constraint allows an alternative to present practice: fixing the free parameter (e.g., the mixing length factor $\alpha$ ) directly by terrestrial experiments and numerical simulations which deal with the process of turbulence itself (Arnett, Meakin, \& Young 2010), instead of calibrating it from complex astro-

${ }^{1}$ This is essentially the "four-fifths law of Kolmogorov", Frisch (1995), p. 76. nomical systems (stellar atmospheres) as is now done.

Second, there has been a considerable development in understanding the nature of chaotic behavior in nonlinear systems; see Cvitanović (1989) for a review and reprints of original papers, and Frisch (1995); Gleick (1987); Thompson \& Steward (1986). Lorenz (1963) presented a simplified solution to the Rayleigh problem of thermal convection (Chandrasekhar 1961) which captured the seed of chaos in the Lorenz attractor, and contains a representation of the fluctuating aspect of turbulence not present in MLT. This advance was allowed by the steady increase in computer power and accessibility, which lead to the exploration of solutions for simple systems of nonlinear differential equations (see Cvitanović (1989) and references therein). It became clear that the Landau picture (Landau 1944) of the approach to turbulence was incorrect both theoretically (Ruelle \& Takens 1971) and experimentally (Libchaber \& Mauer 1982). A striking feature of these advances has been the use of simple mathematical models, which capture the essence of chaos in a model with much reduced dimensionality compared to the physical system of interest.

Third, it has become possible to simulate turbulence on computers. This realizes the vision of John von Neumann (von Neumann 1948), in which numerical solutions of the Navier-Stokes equations by computer are used to inform mathematical analysis of turbulence. In this paper we will follow this idea of von Neumann, in the style which proved successful for chaos studies: building simple mathematical models of a more complex physical system (in this case, the numerical simulations of turbulent convection). This approach should lead to algorithms suitable for implementation into stellar evolution codes, which, unlike MLT, are (1) based upon solutions to 
fluid dynamics equations, (2) non-local, (3) time-dependent, and (4) falsifiable by terrestrial experiment and future improved simulations.

Our particular example is a set of simulations of oxygen burning in a shell of a star of $23 \mathrm{M}_{\odot}$ (Meakin \& Arnett 2007b). This is of astronomical interest in its own right as a model for a supernova progenitor, but also happens to represent a relatively simple and computationally efficient case, and has general implications for the convection process in all stars.

Three-dimensional hydrodynamic simulations of shell oxygen burning exhibit bursty, recurrent fluctuations in turbulent kinetic energy (Meakin \& Arnett (2007b) and below). The reason for this behavior has not been explained theoretically. These simulations show a damping, and eventual cessation, of turbulent motion if we artificially turn off the nuclear burning (Arnett, Meakin. \& Young 2009). Further investigation (Meakin \& Arnett 2009) shows that nearly identical pulsations are obtained with a volumetric energy generation rate which is constant in time, so that the cause of the pulsation is independent of any temperature or composition dependence in the oxygen burning rate. Localized heating is necessary to drive the convection; even with this time-independent rate of heating, pulses in the turbulent kinetic energy still occur.

Such behavior is fundamentally different from traditional nuclear-energized pulsations dealt with in the literature (e.g., the $\varepsilon$-mechanism, Ledoux (1941); Unno, et al. (1989)), and is a consequence of time-dependent turbulent convection (it might be called a " $\tau$ mechanism", with $\tau$ standing for turbulence). It appears to be relevant to all stellar convection. Woodward, Porter, \& Jacobs (2003) found, in a very different context, that nonlinear interaction of the largest modes excited pulsations of a red-giant envelope 2 , which is another example of the $\tau$-mechanism.

In Section 2 we examine the the physics context of the turbulence, including implications of subgrid and turbulent dissipation for the implicit large eddy simulations (ILES) upon which our analysis is based, and the effect of the convective Mach number on the nature of the flow. In Section 3 we review the 3D numerical results of shell oxygen burning which are relevant to the theory. In Section 4 we present the results of the classical Lorenz model (Lorenz 1963) for conditions similar to those in Section 3. In Section 5 we consider implications of turbulent intermittency on stellar variability, and provide a model light curve from this effect alone. Section 6 summarizes the results. The appendix gives a short derivation of the Lorenz model.

\section{Physics Context.}

In this section we summarize concepts which are needed for the interpretation of later results.

\subsection{Subgrid Damping and Kolmogorov}

Approximation of partial differential equations by discrete methods inevitably leads to a loss of information at scales smaller than the grid size. A single element in space is approximated as a homogeneous entity 3 ; this is equivalent to complete mixing at this scale, at each time step, of mass, momentum, and energy. The loss of information that occurs with this mixing corresponds to an increase in entropy (Shannon 1948), the mixing of momentum is

\footnotetext{
${ }^{2} \mathrm{~A} \kappa$-mechanism, which depends upon variations in opacity, is not required to drive such pulsations.

${ }^{3}$ Actually most modern simulations (ours included) use higher order methods which make some further assumptions regarding the behavior of variables inside a zone. This complicates but does not change the argument; it is still true that information is lost at the zone level.
} 
equivalent to the action of viscosity, and the mixing of internal energy corresponds to the transport of heat (Landau \& Lifshitz (1959), $\S 15$ and $\S 49)$.

In 3D flow, turbulent energy will cascade from large scales to small, at a rate set by the largest scales (Kolmogorov 1941). At sufficiently small scales, microscopic processes homogenize the flow and dissipate the kinetic energy. Thus, there is a deep connection between the turbulent cascade and sub-grid scale mixing.

Sytine, et al. (2000) have demonstrated that the piece-wise parabolic method (PPM, which we use), based on the Euler equation (which has no explicit viscosity), converges to the same limit as methods based on NavierStokes equation (which do have explicit viscosity), as the grid is refined to smaller zones and smaller effective viscosity (the relevant limit for astrophysics). The subgrid scale dissipation for monotonicity preserving hydrodynamic algorithms (Boris 2007; Woodward 2007), which is implicit in these methods, automatically gives a reasonable treatment of the turbulent cascade down to the grid scale. We use this implicit sub-grid dissipation in our large eddy simulation (ILES); this is the most computationally efficient way to deal with turbulent systems with a large range of scales. The largest scales, which set the rate of cascade and contain most of the energy are explicitly calculated, while the sub-grid scales are dissipated in a way consistent with the Kolmogorov cascade.

Woodward et al. (2006) have presented a refinement of the PPM algorithm which has improved behavior at the smaller resolvable scales. We have not yet implemented this modification. The theoretical approach used here involves integrated properties of convective cells; we find by direct resolution studies that these properties are well estimated even with surprisingly modest resolution, be- cause they are determined primarily by the largest scales in the convective region. It appears that the ILES simulations are adequate for the present analysis.

Arnett, Meakin, \& Young (2009) have shown that the numerical damping at sub-grid scales in our ILES simulations is quantitatively consistent with the introduction analytically of the Kolmogorov cascade into the theoretical discussion. The turbulent velocity field was found to be dominated by two components:

1. a non-isotropic flow of the largest scale modes in the convection zone, which is coupled to the fluctuations. This has aspects of a "coherent structure" (Holmes, Lumley, \& Berkooz 1996). The largest scales are unstable toward breakup, but are least affected by dissipation, and in this sense the most laminar.

2. a more isotropic, homogeneous turbulent flow which carries the kinetic energy via the turbulent cascade to scales small enough for dissipation to occur (Kolmogorov 1962). Because of the vast size difference, the small scales are weakly coupled to the largest scales, which determine the rate at which energy flows through the cascade.

If we approximate the non-isotropic component of the flow (the largest scale of convection) with that described by the Lorenz model, this interpretation captures the oxygenburning fluctuations.

\subsection{Types of Flow}

There are two limiting cases for convective flow, depending upon the convective Mach number $\mathcal{M}_{\text {conv }}$ (the ratio of the fluid speed to the local sound speed); these are usually termed the "incompressible" $\left(\mathcal{M}_{\text {conv }} \ll 1\right)$ and "compressible" $\left(\mathcal{M}_{\text {conv }} \sim 1\right)$ regimes. 
Stars are stratified in density, so that the notion of "incompressiblity" is misleading. We will use the term "low-Mach number flow" in place of "incompressible" when we mean flows in which acoustic radiation is small, but may be compressed due to stratification.

For turbulent motion, the pressure perturbation $P^{\prime}$ is related to the convective Mach number by $P^{\prime} / P \sim \rho u_{r m s}^{2} / P \sim \mathcal{M}_{\text {conv }}^{2}$. Sound waves outstrip fluid motion, so that pressure differences quickly become small, except possibly for a static background stratification. Most of the historical research on convection (e.g., the Bènard problem, Chandrasekhar (1961); Landau \& Lifshitz (1959)) is done in this limit.

Using the Reynolds decomposition, $\varphi=$ $\varphi_{0}+\varphi^{\prime}$, with horizontal averaging $\langle\varphi\rangle=\varphi_{0}$ and $\left\langle\varphi^{\prime}\right\rangle=0$, mass conservation for a steady state flow can be written

$$
\langle\rho \mathbf{u}\rangle=\left\langle\rho_{0} \mathbf{u}_{\mathbf{0}}\right\rangle+\left\langle\rho^{\prime} \mathbf{u}^{\prime}\right\rangle=0 .
$$

The Navier-Stokes equation, using mass conservation, is

$$
\partial_{t} \rho \mathbf{u}+\nabla \cdot \rho \mathbf{u u}=-\nabla P+\rho \mathbf{g}+\nu \nabla^{2} \mathbf{u}
$$

where $\rho \mathbf{u u}$ is the Reynolds stress tensor. Mass conservation implies a convenient identity involving total co-moving derivatives,

$$
D_{t} \rho \mathbf{u}=\rho D_{t} \mathbf{u} .
$$

Taking the dot product of the velocity vector $\mathbf{u}$ with the Navier-Stokes equation, gives a kinetic energy equation,

$$
D_{t} \rho \mathbf{u} \cdot \mathbf{u} / 2=-\mathbf{u} \cdot \nabla P+\rho \mathbf{u} \cdot \mathbf{g}+\nu \mathbf{u} \cdot \nabla^{2} \mathbf{u} .
$$

If on average the system is in a steady state, the time derivative must integrate to zero over the convective region, and the mass conservation law implies that the total buoyancy power term is zero, $\langle\rho \mathbf{u} \cdot \mathbf{g}\rangle=0$ (assuming constant $\mathrm{g}$ on horizontal averaging), and therefore does not contribute to the production of kinetic energy anywhere in the flow. The only other term, which remains to balance the viscous dissipation of the kinetic energy, is the pressure term

$$
\mathbf{u} \cdot \nabla P=\mathbf{u}_{\mathbf{0}} \cdot \nabla P_{0}+\left\langle\mathbf{u}^{\prime} \cdot \nabla P^{\prime}\right\rangle .
$$

which may be rewritten as

$$
\mathbf{u} \cdot \nabla P=\mathbf{u}_{\mathbf{0}} \cdot \nabla P_{0}+\left\langle\nabla \cdot\left(P^{\prime} \mathbf{u}^{\prime}\right)\right\rangle-\left\langle P^{\prime} \nabla \cdot \mathbf{u}^{\prime}\right\rangle .
$$

The divergence term vanishes upon integration over the volume. Using hydrostatic equilibrium for the background state, $\nabla P_{0}=\rho_{0} \mathbf{g}$, and mass conservation, $\rho_{0} \mathbf{u}_{\mathbf{0}}=-\left\langle\rho^{\prime} \mathbf{u}^{\prime}\right\rangle$,

$$
\langle-\mathbf{u} \cdot \nabla P\rangle=\left\langle\rho^{\prime} \mathbf{u}^{\prime} \cdot \mathbf{g}\right\rangle+\left\langle P^{\prime} \nabla \cdot \mathbf{u}^{\prime}\right\rangle
$$

When the Mach number is small, the second term the right hand expression is nearly zero because $\nabla \cdot \mathbf{u}^{\prime} \approx \mathbf{0}$ and the turbulent pressure is negligible. In this limit the kinetic energy production is best understood as due to the remaining buoyancy power term $\left\langle\rho^{\prime} \mathbf{u}^{\prime} \cdot \mathbf{g}\right\rangle$, which is directly related to the enthalpy flux (Arnett, Meakin, \& Young 2009).

When the Mach number is no longer small, the second term on the RHS of Eq. 8 increases in importance: both the divergence of the fluctuating velocity field and the pressure perturbation begin to play a role. The velocity field changes character; it is no longer dominated by rotational flow, but develops an irrotational component $\left(\nabla \cdot \mathbf{u}^{\prime} \neq 0\right)$. The flow becomes diverging (consider the extreme limit of a point explosion which is pure divergence). Also, the ram "pressure" (a tensor $\rho \mathbf{u u}$ ) is not negligible and must be included in the momentum equation ("hydrostatic equilibrium"). Sound wave generation increases rapidly as $\mathcal{M}_{\text {conv }} \rightarrow 1$ (Landau \& Lifshitz (1959), §75). The compressible limit is $\mathcal{M}_{\text {conv }} \simeq 1$. Shock formation is the most startling change in the flow character. 
Which of the $\mathcal{M}_{\text {conv }}$ limits is relevant for astrophysics? Both are. Almost all the matter in stellar convection zones, during almost all evolution, is in the limit of low-Mach number flow, as are our turbulence simulations. The exceptions are important: (1) explosions, such as supernovae and novae, (2) vigorous thermonuclear flashes, (3) vigorous pulsations, especially radial ones, and (4) the sub-photospheric layers of stellar surface convection zones, which are strongly nonadiabatic, to name a few.

\section{The Oxygen Shell Simulation}

Figure1 1llustrates the behavior of two important integral quantities, the total turbulent kinetic energy and the total buoyancy power, in the oxygen-burning shell simulations for a $23 M_{\odot}$ star (Meakin \& Arnett 2007a). The flow has a low-Mach number $\left(\mathcal{M}_{\text {conv }}^{2} \leq 0.001\right)$, although the numerical simulations use the equations for fully compressible fluid flow, and would have correctly treated high-Mach number flows. Also shown are the same quantities for the Lorenz model, which is discussed in Section 4 .

At any instant in time, the total convective kinetic energy is $\frac{1}{2} M_{C Z} v_{r m s}^{2}$, where $M_{C Z}$ is the mass of the convective zone and $v_{r m s}$ is the rms velocity 4 , while the kinetic energy in the isotropic part of the turbulent flow field is $E_{\text {turb }}=\frac{1}{2} M_{C Z} u_{t}^{2}$, where $u_{t}$ is the turbulent velocity, which we define as the isotropic part of the turbulent flow5. The reader is warned

\footnotetext{
${ }^{4}$ More precisely, $v$ is the solenoidal velocity in the convective region, with overall translational velocity removed, so that $v_{r m s}$ is its root-mean-square value.

${ }^{5}$ This follows Arnett, Meakin, \& Young (2009), §2.4, in which the transverse velocities are used to estimate the isotropic component of the vertical velocity. Thus, we use $u_{t}^{2}=\frac{3}{2}\left(u_{x}^{2}+u_{y}^{2}\right)=\frac{3}{4} v_{r m s}^{2}$, where $u_{x}$ and $u_{y}$ are the velocity components perpendicular to the direction of the gravity vector, i.e., the tangential velocities. The vertical velocity also contains a significant contribution $\left(\frac{1}{4} v_{r m s}^{2}\right)$ from the non-isotropic flow of the
}
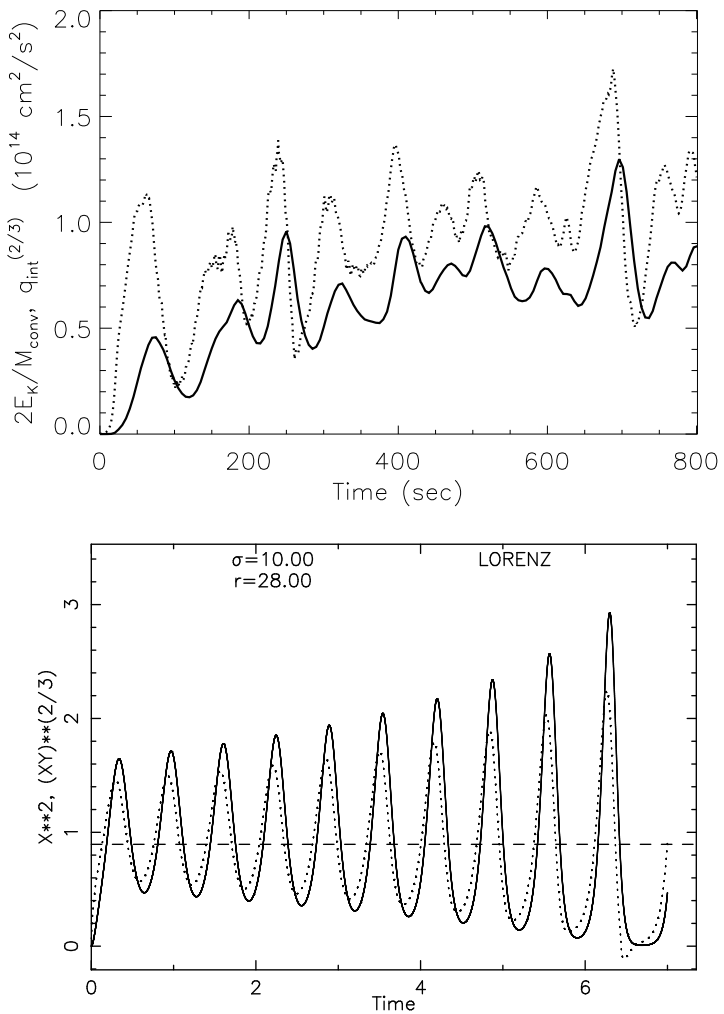

Fig. 1.- Comparison of Convective Kinetic Energy Fluctuations in the 3D simulations and in the Lorenz model, starting from zero velocity. The Lorenz model is labeled with its Prandtl number $\sigma$ and its Rayleigh number $r$. Turbulent velocity squared (solid) and buoyancy power to the $2 / 3$ power (dotted) are plotted versus time. The axes where chosen so that the same number of peaks would be shown, and the average kinetic energy be the same. The curves are surprisingly similar, even though (1) the Lorenz model used only a single mode, (2) $\sigma$ and $r$ were not adjusted to give a fit, (3) the Lorenz model imposes thermal balance while thermal imbalance adds to the background slope in the simulation (see Fig. 2), and (4) the simulations have $\sim 10^{8}$ more degrees of freedom than the Lorenz model. 
that division of the flow into "turbulent" and "large scale" flow is useful but an oversimplification, so that the exact relative values of these two kinetic energies depend upon the algorithm used in their definition, but is of order unity Arnett. Meakin, \& Young 2009; Meakin \& Arnett 2011). Consequently the precise distinction does not change the qualitative picture.

The buoyancy power is the rate at which kinetic energy per unit mass is increased by buoyant acceleration. If it is integrated over the space containing the convection zone, we have $q_{\text {int }}=\int_{C Z} q d r$, where $q=-g u^{\prime} \rho^{\prime} / \rho_{0}$ is the buoyant acceleration times the turbulent velocity; $q_{\text {int }}$ has units of velocity cubed.

In Figure 1 the simulations show a phase lag of about 20 seconds between the peaks in buoyancy term $\left(q_{\text {int }}^{2 / 3}\right)$ and turbulent kinetic energy. This is about half the time it takes the flow to transit a distance $\ell_{C Z}$, the depth of the convection zone. It also corresponds to an e-folding time for turbulent kinetic energy decay due to Kolmogorov damping, where $\varepsilon_{K}=u_{r m s}^{3} / \ell_{d}=\left(u_{r m s}^{2} / 2\right)\left(2 u_{r m s} / \ell_{d}\right)$. Power spectra for both variables peak at 89 seconds; an average transit time is $51 \mathrm{~s}$.

Figure 1 shows multimodal behavior in the 3D simulations; preliminary results from a quantitative analysis Meakin \& Arnet 2011) using principal orthogonal decomposition (POD) indicates that a single dominant mode has about $43 \%$ of the kinetic energy, the first five modes have $75 \%$, and $90 \%$ is reached with the eleventh mode. There is a strong dominant mode but also significant energy in several other modes; the modes interact in a nonlinear and dynamic way.

The buoyancy power is a large scale feature and is strongly anisotropic (plumes move

largest eddies. A more careful discussion of the velocities in terms of principal orthogonal decomposition is in preparation. vertically). The dissipation implied by the turbulence occurs at the Kolmogorov scale (which is tiny); this dissipation is wide-spread in space (Arnett, Meakin, \& Young 2009), including the entire turbulent region on average, and bounded by the stably stratified layers. Because buoyancy and dissipation occur at vastly different length scales, they are weakly coupled.

In low Mach number flow, on average over time, the buoyant driving must balance the turbulent damping for a quasi-steady state to exist (see Arnett, Meakin, \& Young (2009) for a detailed discussion, especially their Eq. 33). The time averaged viscous dissipation is

$$
\epsilon_{\text {turb }}=\overline{q_{\text {int }}} / \ell_{C Z} \approx \overline{v_{r m s}^{3}} / \ell_{d} \approx 1.54 \overline{u_{t}^{3}} / \ell_{d},
$$

where $\ell_{d}$ is the dissipation length, $\ell_{C Z}$ is the depth of the convection zone, and the over-lines indicate an average over time (two turnover times or four transit times, about 200 seconds, in the analysis).

From Equation 9 it is clear that the turbulent dissipation is of third order in the turbulent velocity, while the buoyancy power, $q=-g u^{\prime} \rho^{\prime} / \rho_{0}$, is second order (because $\rho^{\prime}$ is first order). This means that in the turbulent regime there is a unique turbulent velocity which satisfies the condition that buoyancy power balance turbulent damping. This has the nature of an eigenvalue problem. The assumption of a turbulent cascade implies finite turbulent velocities, with macroscopic structure. Equation 9 applies as an integral over a region much larger than the Kolmogorov scale; at the Kolmogorov scale microscopic dissipation occurs by the Navier-Stokes term (Landau \& Lifshitz (1959), §15). If we dot this Navier-Stokes term with the velocity to generate a rate of kinetic energy destruction by viscosity (see Equation 5), we have

$$
(\mathbf{u} \cdot \partial \mathbf{u} / \partial t)_{v i s c}=\nu \mathbf{u} \cdot \nabla^{2} \mathbf{u}
$$


which does scale as the velocity to second order. When integrated over the turbulent cascade, Equation 10 becomes Equation 9, The extra factor of velocity comes from the rate at which turbulent kinetic energy is delivered to the Kolmogorov scale. Turbulence is an inherently nonlinear process, which sets up its own scales and structures. Care must be taken in analyzing it with techniques involving expansion in series 6 .

The dissipation is seen to be driven by the isotropic part of the flow $u_{t}$, which accounts for about three-fourths of the kinetic energy according to estimates by Arnett, Meakin, \& Young (2009) (which are roughly similar to those from POD analysis by Meakin \& Arnett (2011)). Note that $v_{r m s}^{2}=\frac{4}{3} u_{t}^{2}$ which gives the vertical offset between the solid and dotted curves in Figure 1. Consider the average conditions from 200 to 800 seconds in the simulations. The average level of buoyancy (actually $q_{\text {int }}^{2 / 3}$ is plotted) is higher than that of kinetic energy in isotropic turbulence. If we equate (1) the power generated by buoyancy, to (2) the dissipation due to turbulence, both averaged over a few transit times, we find

$$
\ell_{d} / \ell_{C Z}=\overline{u_{r m s}^{3}} / \overline{q_{i n t}} \approx 0.85 .
$$

Thus, in the Meakin \& Arnett (2007b) simulations, the dissipation length is found to be essentially the depth of the convective zone, consistent with Kolmogorov theory.

These results are far more general than the particular stellar situation we have discussed. While the neutrino cooling may seem exotic to some stellar evolutionists, in fact it behaves somewhat like the more familiar cooling by radiative diffusion, and has no strange effect on the turbulence. We note that the original simulations (Meakin \& Arnett 2007b), which

${ }^{6}$ See Arnett \& Meakin (2011b) for an example of the danger of using linear stability analysis for turbulence in stars. included core hydrogen burning cooled by radiative diffusion as well as oxygen shell burning cooled by neutrinos, explicitly showed this similarity.

In the long term, the thermal state of the convection zone is supposed to evolve toward a global thermal balance between total heating by nuclear burning and total cooling by neutrino emission (Arnett 1972, 1996). This is illustrated in Figure 2, which shows time average luminosities of heating and of cooling, as a function of the entropy of the convection zone. At lower entropy, as in the $3 \mathrm{D}$ simulation, the heating is dominant, causing the entropy to increase. This is accomplished primarily by expansion, with a small increase in temperature. The decrease in density causes a bigger change in nuclear burning than in neutrino cooling, so that a thermal balance would be attained (shown as a vertical line) when the heating and cooling curves cross.

The gradual rise in turbulent kinetic energy in the simulation, shown in Figure 1, occurs in a shell which is below the entropy of balanced heating and cooling, so that heating dominates. The pulses are much faster than this secular evolution, which changes on a time scale of $t \geq 10^{3}$ seconds.

\section{The Lorenz Solution}

The Lorenz model is a convective roll, or cell, whose dynamics are described by three amplitude equations. This is a simple example of an elegant method of reduction of turbulent flow to a low-order set of dynamical equations using amplitudes of a proper orthogonal decomposition (POD) of numerical simulations or extensive experimental data sets (Holmes, Lumley, \& Berkooz 1996).

The Lorenz model is a better mathematical representation of the dynamics of a convective cell than MLT in that the acceleration and deceleration over a convective cy- 
cle are integrated to determine the motion, rather than prescribed. Because it uses a single mode, the Lorenz model does not have sensitivity to local variation; it is a global model, unlike MLT, which is local. Lorenz devised the model as a point of principle test of meteorological convection, which like the stellar problem, is damped by a turbulent cascade. To make use of this extensive literature (Cvitanović 1989; Frisch 1995; Gleick 1987; Thompson \& Steward 1986), we use the original version of Lorenz (1963), with the same Prandtl and Rayleigh numbers, to explore the implications on dynamics of the strange attractor. The original Lorenz formulation is a 2D, low Mach number, and single mode model (equivalent to Figure [3). A transition to 3D may be made using the solutions of Chandrasekhar (1961), but is not necessary for present purposes. Real convection (Libchaber \& Mauer 1982) is expected to be single mode only near the onset of convective instability.

The Lorenz equations (see Appendix A) are:

$$
\begin{array}{rlrl}
d X / d \tau= & & -\sigma X+\sigma Y \\
d Y / d \tau & = & -X Z+r X-Y \\
d Z / d \tau= & X Y-b Z,
\end{array}
$$

In the classical Lorenz model, $X$ is proportional to the speed of convective motion, $Y$ is proportional to the temperature difference between ascending and descending flow, and $Z$ is proportional to the distortion of the vertical temperature profile from adiabatic (Lorenz 1963). Here $\tau$ is a time in thermal diffusion units, $\sigma$ is the effective Prandtl number, $r$ is the ratio of the Rayleigh number to its critical value for onset of convection, and $a$ is the ratio of the depth to the width of the convective cell, so that $b=4 /\left(1+a^{2}\right)$; see Lorenz (1963). The Prandtl number is the ratio of coefficients of the viscous dissipation term to the thermal

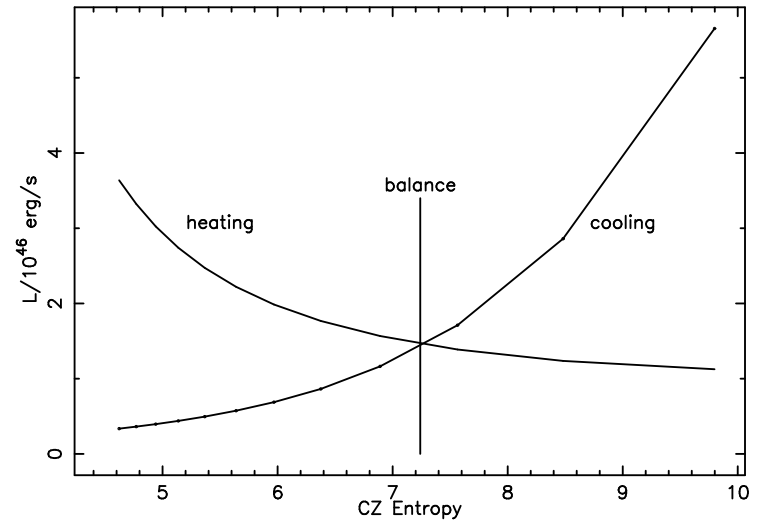

Fig. 2.- Nuclear heating and neutrino cooling in an oxygen burning shell, as a function of entropy. At lower entropy, as in the simulations, nuclear heating dominates because of its density dependence. For the same temperatures but higher entropy, the inhibition of neutrino-antineutrino emission is reduced. The shell entropy slowly increases until cooling balances heating, at which point thermal balance is attained.

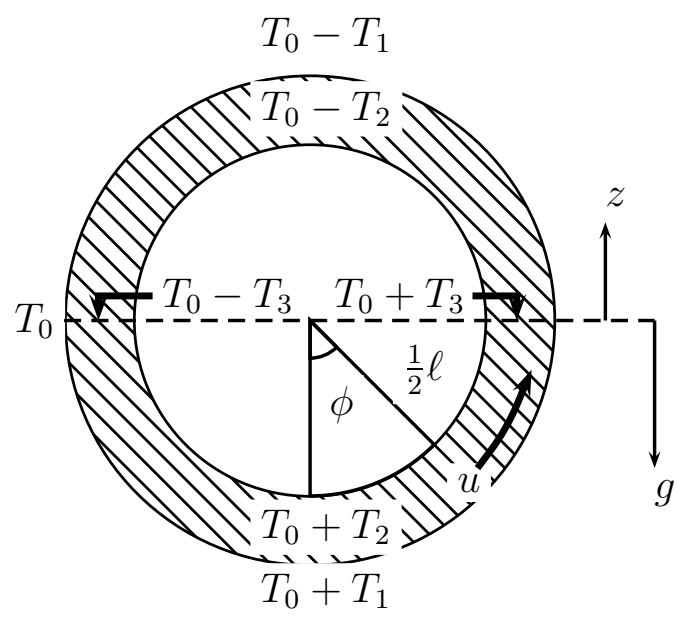

Fig. 3.- The Lorenz Model of Convection: Convection in a Loop. 
mixing term. Lorend 7 took $\sigma=10$, and chose the most unstable mode so that $a^{2}=1 / 2$, and $b=8 / 3$. For this mode, and a Rayleigh number of $r=470 / 19=24.74$ times the critical value, steady flow becomes unstable.

Figure 3 illustrates the structure and notation in the Lorenz model. The Prandtl number may be expressed as $\sigma=\tau_{\text {rad }} / \tau_{v i s c}$, the ratio of the radiative cooling time scale to the viscous time scale. The viscous damping time is taken to be constant, $\tau_{v i s c}=1 / \Gamma$. The Rayleigh number, in units of its value at the onset of laminar convection, is

$$
r=g \beta_{T} T_{1} / \ell \Gamma K T_{0},
$$

where $K=1 / \tau_{\text {rad }}, \beta_{T}=-(\partial \ln \rho / \partial \ln T)_{P}$, and $g$ is the gravitational acceleration. The lapse rate of the adiabatic background $T_{E}$ is $g / C_{P}$, so $T_{1}=g(\ell / 2) / C_{P}$. In astrophysical notation,

$$
\nabla-\nabla_{a}=\nabla_{a}\left(T_{2} / T_{1}-1\right)
$$

where $T_{2}$ is the temperature variation in the vertical direction (Fig. 3). The depth of the roll, in pressure scale heights, is

$$
\ell / H_{P}=\frac{1}{\nabla_{a}} \ln \left[\left(T_{0}+T_{1}\right) /\left(T_{0}-T_{1}\right)\right] .
$$

\subsection{Energy fluxes}

In the meteorological case the fluid motion (wind) is of primary interest, however the energy fluxes 8 provide a means to connect with stellar observations.

\footnotetext{
${ }^{7} \mathrm{~A}$ justification is simply that this allows the three Lorenz equations to capture chaotic behavior. See Appendix $\mathrm{B}$ for a discussion of the physical implications of the Prandtl number for stars.

${ }^{8}$ In discussion of the Lorenz model, both heat flux and enthalpy flux are mentioned; which is correct? Depending upon the physics perspective, both may be. In the simplest Lorenz model, the fluid in strictly incompressible, so that the work term $P d V$ is strictly zero, and in this case only heat content can carry energy, so heat flux is relevant. However, we may inter-
}

The vertical enthalpy flux due to radiative diffusion of the background is

$$
\begin{aligned}
F_{E} & =-\rho \nu_{T} C_{P} d T_{E} / d z \\
& =\rho \nu_{T} C_{P} T_{1}(2 / \ell)
\end{aligned}
$$

which is constant in the Lorenz model. The thermal conduction coefficient is $\nu_{T}=$ $\left(4 a c T^{3} / \rho \kappa\right) /\left(\rho C_{P}\right)$ and $K=\nu_{T}(1 / \ell)^{2}$. Here $z=-\frac{\ell}{2} \cos \phi$, the radius of the roll being $\ell / 2$. Because the background temperature is taken to be linear in $z$, the divergence of this flux is identically zero, and therefore gives no local heating or cooling. The additional vertical enthalpy flux, due to the temperature perturbation, is

$$
\begin{aligned}
F_{z} & =-\rho \nu_{T} C_{P} d\left(T-T_{E}\right) / d z \\
& =\rho \nu_{T} C_{P}\left(T_{2}-T_{1}\right)(2 / \ell) .
\end{aligned}
$$

If we define a potential temperature $9 T_{4}=$ $T_{1}-T_{2}$, the vertical flux is separated into two parts, so that the adiabatic background value is denoted by $T_{1}$ and the changes caused by motion are contained in $T_{4}$.

The flux in the horizontal direction is

$$
\begin{aligned}
F_{y} & =-\rho \nu_{T} C_{P} d T / d y \\
& =-\rho \nu_{T} C_{P} T_{3}(2 / \ell),
\end{aligned}
$$

which averages to zero by symmetry; here $y=$ $\frac{\ell}{2} \sin \phi$. Both $F_{z}$ and $F_{y}$ are proportional to a potential temperature which varies in time.

The net vertical enthalpy flux will not be constant in height $z$, so that its divergence is nonzero. This implies local heating/cooling,

pret the equations in terms of a stratified system with low-Mach number flow, having a stratification in density as well as temperature. Then $d V$ is not zero, $P d V$ work is done, and the relevant flux is the enthalpy flux.

${ }^{9}$ Here potential temperature is used as in fluid dynamics and meteorology (Dutton 1986; Tritton 1988); the potential temperature is measured relative to an adiabatic background, and may be small even if the convective region is strongly stratified (as is often the case in stars). 
which would have to be compensated for in a steady state, and is a consequence of considering only a single mode. Smaller scale modes would be needed to deal with the local heating/cooling; see Canuto \& Mazzitelli (1991); Canuto et al. (1996) for efforts to include a full spectrum of modes. To get the average flux through the cell for a single mode, we take the double projection, of $u$ and of $T$ on the vertical direction,

$$
\begin{aligned}
F_{e}(\phi) & =\rho u_{z} C_{P} T \\
& \rightarrow \rho C_{P} u T_{3} \sin ^{2} \phi .
\end{aligned}
$$

The enthalpy flux is zero at the top and bottom of the roll $(\phi=\pi$ and 0$)$, and a maximum at the midpoint $(\phi=\pi / 2)$. The average value of $\sin ^{2} \phi$ over the roll, $\phi=0$ to $2 \pi$, is 0.5. This is to be compared with the $\alpha_{E}$ of Arnett, Meakin, \& Young (2009), which was the range 0.68 to 0.85 for the simulations then available. Because the simulations are multimode, this slightly higher value for the correlation seems natural.

Integrating over the cell, $0 \leq \phi \leq 2 \pi$, gives

$$
F_{e}=\frac{1}{2} \rho C_{P} u T_{3}
$$

which is the vertically averaged enthalpy flux of the cell. This is the "convective flux" in stellar models. The up-flow enthalpy flux equals the down-flow value, and both are positive (hot up-flows and cold down-flows both transport energy upward).

The ratio of enthalpy flux to the total radiative flux $\left(F_{z}+F_{E}\right)$ is

$$
F_{e} / F_{r}=\left(\ell / 4 \nu_{T}\right)\left(u T_{3} / T_{2}\right)
$$

which is a function of time.

The kinetic flux exactly cancels in this formulation, as in MLT, with the up-flow being the negative of the down-flow. This is not true in the simulations. The kinetic flux does approach zero for convective regions which are so thin that they are almost unstratified. However, stratified convection zones have an asymmetry in up and down flows, giving a modest net kinetic energy flux (downward for pure top driving, up for pure bottom driving, and both for more general cases, Meakin \& Arnett (2009)).

We may express fluxes in units of the radiative flux of the background, $F_{E}$, and in terms of the variables in the Lorenz equations. The excess vertical radiative flux is

$$
\begin{aligned}
F_{z} / F_{E} & =T_{4} / T_{1} \\
& =Z / r
\end{aligned}
$$

where $T_{1}=g \ell / 2 C_{P}, T_{0}=g H_{P} /\left(C_{P}-C_{V}\right)$ and $\gamma=C_{P} / C_{V}$, and

$$
r=\frac{\gamma-1}{2 \gamma}\left(\frac{g Q}{H_{P} K \Gamma}\right) .
$$

The horizontal radiative flux is

$$
\begin{aligned}
F_{y} / F_{E} & =T_{3} / T_{1} \\
& =Y / r .
\end{aligned}
$$

The enthalpy flux of the cell, in the vertical direction, is

$$
F_{e} / F_{E}=X Y / 2 r .
$$

In general, without need for any additional mechanism to cause variability, the net flux of energy through a turbulent cell varies with time.

\subsection{Steady-state solutions}

A steady state solution for the Lorenz equations is

$$
X=Y=Z=0,
$$

and, for $r \geq 1$, a second solution, for a stable convective roll, appears:

$$
X=Y= \pm[b(r-1)]^{1 / 2} ; Z=r-1 .
$$


The second solution is unstable if $\sigma>2$ and $r>r_{c}$ where

$$
r_{c}=\sigma(\sigma+b+3) /(\sigma-b-1),
$$

and this instability gives rise to fluctuations in velocity (Lorenz 1963). The instability also gives fluctuations in energy flow through the cell.

The steady state solutions might be of practical value for stellar evolutionary codes, to the extent that they provide an estimate of the average behavior of the convective cell. However, the fluctuations are large (in no sense are they "small perturbations"), and may cause nonlinear complications. For example, a thermonuclear runaway would be sensitive to the largest value of the temperature fluctuations because that would affect the burning rate (Arnett \& Meakin $2011 \mathrm{~b})$. The fluctuations drive entrainment episodes, and affect the mixing of composition (Meakin \& Arnett 2007b). The fluctuations may be able to modify the driving of pulsations in stars with vigorous convection zones. In red supergiants, coupling of turbulent fluctuations in the surface convection zones to both radial and non-radial pulsations seems likely (see below).

\subsection{Non-steady Solutions}

In order to compare the Lorenz model to $3 \mathrm{D}$ simulations, it is desirable to have comparable starting conditions. Arbitrary choices can give large initial transients before the attractor controls the behavior. The 3D simulations start with zero velocity but finite temperature deviations from an adiabatic gradient. We take

$$
X=0, Y=[b(r-1)]^{\frac{1}{2}}, Z=r-1,
$$

as initial conditions, which sets the velocity to zero and uses steady state values for the temperature fluctuations.
Figure 1 shows the behavior of the Lorenz model of convection (panel labeled LORENZ), for a similar number of pulses as shown for the 3D simulation, and the same variables: buoyancy power $X Y /(b(r-1))$ to the twothirds power, and kinetic energy per unit mass $X^{2} /(b(r-1))$. The factors $b(r-1)$ are chosen so that the steady state values are normalized to unity, but are qualitatively correct for comparison with the $3 \mathrm{D}$ simulations. The velocities in Fig. 1 are not precisely the same, the 3D simulations giving turbulent velocity while the Lorenz speeds are more appropriate to "coherent structures". They are related (see $\S 3.1$ ), as the large scale velocities become unstable and turbulent, and their kinetic energies are the same order of magnitude (Arnett. Meakin, \& Young 2009; Meakin \& Arnett 2011).

The time is measured in the dimensionless units of Lorenz; a turnover time is two transit times, and roughly the time between peaks. We see that the peaks in buoyancy power slightly precede those in kinetic energy, as in the $3 \mathrm{D}$ simulations but less dramatically. This difference is related to the fact that the Lorenz model has viscous dissipation acting directly on the large scale velocities, while the $3 \mathrm{D}$ simulations have dissipation at the Kolmogorov scale, which is separated from the large scale by the turbulent cascade, involving many, many reductions in length scales (see Section B.3). Additional modes would fill in the "valleys" in the Lorenz model (see \$5). Over the time shown, the average kinetic energy is 0.968 of the formal steady state solution, which is shown in Figure 1 as the dashed horizontal line in the Lorenz panel.

The degrees of freedom in the threedimensional simulations, and in the Lorenz model, are dramatically different. The floatingpoint operation count differs by a large factor: $\sim 10^{8}$ (several times $10^{8}$ zones times 7 effective scalar variables for simulations, versus 
three amplitudes for the Lorenz model). With such an extreme compaction, it is striking that they give a similar picture for fluctuations in convection.

\subsection{Long Term Behavior}

Unlike the full 3D simulations, it is trivial to extend the Lorenz model to later times. Figure 4 shows an extension in time by a factor of 10 . There is a relatively steady growth in amplitude up to time $\sim 7$, at which point chaotic behavior begins to appear. The fluctuations in kinetic energy (and $q_{\text {int }}^{\frac{2}{3}}$ ) are large, sometimes exceeding the steady state value $(\sim 1)$ by a factor of 4 . The convective luminosity from a single Lorenz model shows the same qualitative behavior.

This drastic behavior raises two interesting possibilities:

1. The numerical simulations will follow the solutions of the Lorenz equation, and exhibit vigorous and chaotic fluctuations at later times, or,

2. The multimode behavior of the simulations will allow even stronger dissipation, preventing extreme behavior.

Either way, the result is important for the evolution of supernova progenitors, especially regarding the effects of fluctuations on mixing (yields) and outbursts. Simulations of multiple burning shells (C, Ne, O and $\mathrm{Si}$ ) in 2D have been continued further than the 3D simulations for the oxygen burning shell. They appear to follow the first option, so that the prediction from $2 \mathrm{D}$ simulations is that core collapse progenitors will have violent eruptions prior to core collapse (Arnett \& Meakin 2011b). Full 3D simulations need to be extended to later times at which the Lorenz model predicts chaotic behavior.

\subsubsection{Duration}

The oxygen shell might not last for the $\sim 70$ transit times shown in Figure 4, a linear estimate suggests that it consumes its fuel in about 100 traversal times (the Damköhler number is $D_{a} \leq 0.01$, Arnett. Meakin. \& Young $(2009)$ ), so the background evolution should not be neglected for such time intervals. Oxygen burning is likely to be a more dynamic event than previously supposed (e.g., by Woosley, Heger, \& Weaver (2002)). Averaging over multiple cells may moderate the net fluctuation (see below).

For convection zones in other stars, the number of traversal times available may be much larger. The sun has a deep surface convection zone (20 pressure scale heights); a plume would, if unimpeded, fall through the convection zone in about 2 hours; it would take about 7 centuries to process all the mass of the convection zone through the surface layers. These times bracket the effective mixing time, so that of order $10^{7}$ to $10^{13}$ mixing times would occur over the age of the present-day sun.

\subsubsection{Chaotic Behavior}

Figures [5] and 6] show the familiar long term behavior of the Lorenz model (Gleick 1987). Figure 5 shows the trajectory in $\mathrm{X}$ and $\mathrm{Y}$ space for the first 70 dimensionless time intervals. $\mathrm{X}$ is the dimensionless speed and $\mathrm{Y}$ the dimensionless temperature variation in the horizontal direction. Both $\mathrm{X}$ and $\mathrm{Y}$ switch signs, but there is an overall correlation because there is a net enthalpy flux, which is proportional to XY. The correlation is in the sense that hot regions rise and cool regions sink. The radiative flux associated with the horizontal temperature variation is proportional to $\mathrm{Y}$, and while on average over long times is zero, it achieves this in segments of time in which first one then the other direc- 


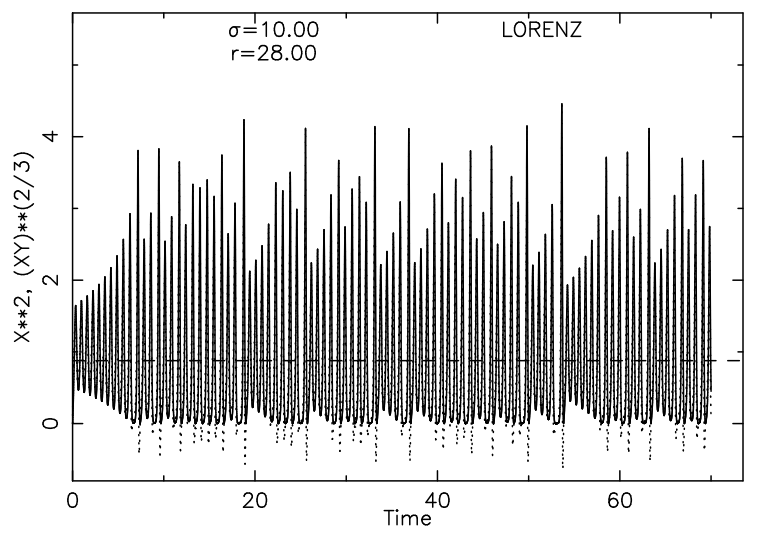

Fig. 4.- Later Behavior of the Lorenz Model of Convection, for $\sigma=10, r=28$, and $b=8 / 3$. The format is the same as Figure 1, but the time interval is longer by a factor of 10. Chaotic behavior is beginning to appear, with fluctuations in kinetic energy and buoyant driving sometimes exceeding the steady state value by a factor of 4 .

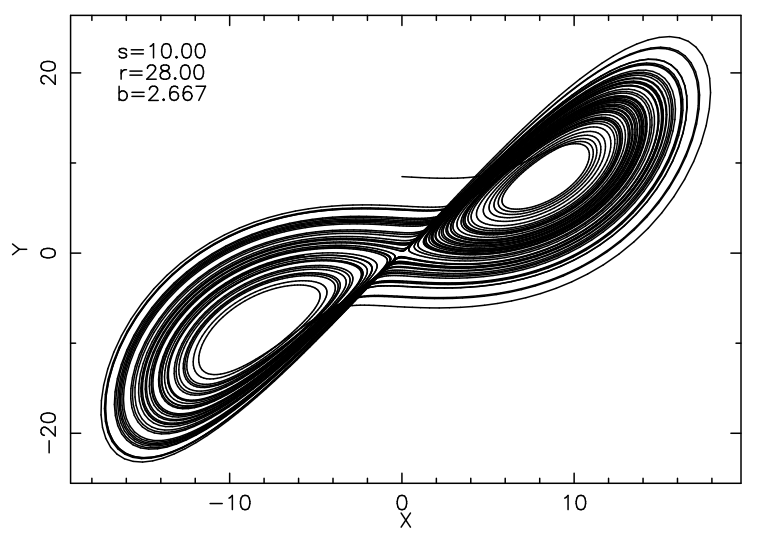

Fig. 5.- Long Term Behavior of the classic Lorenz Model for X (speed) and Y (horizontal temperature fluctuation), for $\sigma=10, r=28$, and $b=8 / 3$. The trajectory is shown for 70 Lorenz time units. Both $\mathrm{X}$ and $\mathrm{Y}$ switch signs, but there is an overall correlation (hot zones rise while cool zones sink). This correlation gives rise to positive values for buoyancy flux and enthalpy flux. tion (sign) are favored. This is the strange attractor at work.

Figure 6 shows the same time interval, but plots $\mathrm{Y}$ and $\mathrm{Z}$ (the dimensionless temperature in the vertical direction, parallel to the gravity vector). Notice that $\mathrm{Z}$ has positive values about which it orbits. The stratification breaks the symmetry in vertical versus horizontal directions. The radiative flux in the vertical direction has a steady part and a fluctuating part. The latter is proportional to Z, and is positive (outward energy flow).

In MLT, the Schwarzschild discriminant $\left(\mathcal{S} \equiv \nabla-\nabla_{a}\right)$ is the temperature excess above adiabatic; this excess is assumed to be proportional to the enthalpy flux. This is inconsistent with the Lorenz model, because the velocity $\mathrm{X}$ can have both signs while $\mathrm{Z}$ does not. The error arises because in MLT the speed of convection is taken to be intrinsically positive (to avoid this problem), and may be traced back to a lack of conservation of mass ("blobs dissolve" into the environment rather than flow back to complete a cycle).

\subsection{3. $M L T$}

What happens if we reduce the three equations of Lorenz to two, forcing one variable to be at its steady state value? This is similar to the MLT approach (assuming the mixing length parameter is chosen so that the kinetic energy scale is physically correct; see Arnett, Meakin, \& Young (2010)). Enforcing the steady state value for the vertical temperature excess, $Z=r-1$, but allowing $X$ and $Y$ to vary, corresponds to a model with a single temperature variable (like MLT). Such integrations are shown in Fig. 7, There are no pulses in kinetic energy or buoyancy; the curves quickly approach a constant value. Convection proceeds by steady motion in a roll; a finite $\mathrm{XY}$ is required to give torque to make the roll. This "two equation model" no longer has a strange attractor; the pulses have 


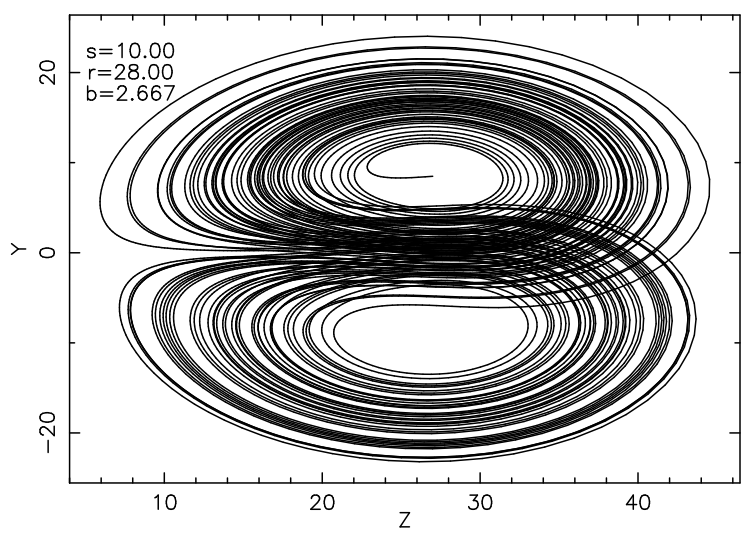

Fig. 6. - Long Term Behavior of the classic Lorenz Model, for $\sigma=10, r=28$, and $b=8 / 3$. Same as Figure [5, but with $\mathrm{Y}$ and $\mathrm{Z}$ (vertical temperature fluctuation) shown. $\mathrm{Z}$ is a positive for these parameters. $\mathrm{Y}$ has both positive and negative steady state values, about which it orbits.

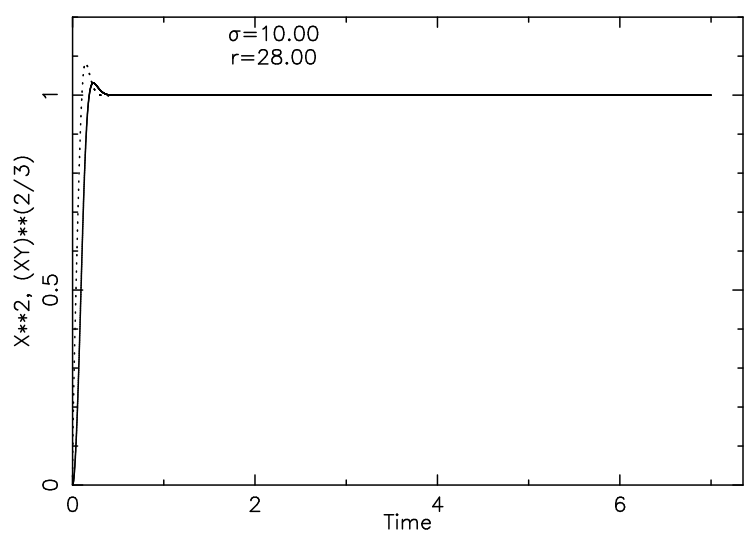

Fig. 7.- Initial Behavior of the two Equation Model of Convection, for $\sigma=10, r=28$, and $b=8 / 3$. The critical value for instability of the convective roll is $r_{c}=24.74$. The format is the same as Figure 1, The turbulent fluctuations are eliminated, as they are in MLT. been eliminated. This explains why stellar evolutionary calculations which use MLT do not show these fluctuation 10 .

\section{Cells and Shells}

\subsection{Multiple Modes in Cascade}

Figure 1 shows that the primary difference in the Lorenz model and the 3D simulations is that the Lorenz model has only a single mode, while the simulations are obviously multimodal. This difference may be superficial. The Lorenz model in this application (as in the original meteorological one) has additional modes implied by the turbulent cascade which mediates the damping (i.e., they are implicitly in $\Gamma$; see Appendices and $(\mathrm{B})$. A simple Richardson cascade was discussed in $\$$ B.3. in which $f$, the fractional change in length scale for each step in the cascade, is assumed to be a constant (Frisch 1995; Davidson 2004). This is not very plausible for the largest scale modes because they are the most sensitive to boundary conditions (they must fit into the convective region), but is simple and instructive. The fractional time spent in the cascade for each mode may be shown to be the fractional kinetic energy in that mode. Using $\$$ B.3. this gives $f^{(2 / 3)(n-1)}\left(1-f^{2 / 3}\right)$ for $n=1,2, \cdots$, or roughly $0.37,0.23$, and 0.14 for the first three. There is a dominant mode accompanied by several weaker but significant ones.

One way to proceed would be to introduce additional modes into the Lorenz model (chosen with guidance by the 3D simulations), and to generate an expanded set of amplitude equations which generalize the three of Lorenz (Eq. 12, 13, and 14). This would make the system multimodal, and allow for modal interac-

${ }^{10}$ In analogy, the two-body (Kepler) problem in celestial mechanics is well behaved, while the unrestricted three-body problem is far more complex (Poincaré 1893 ). 


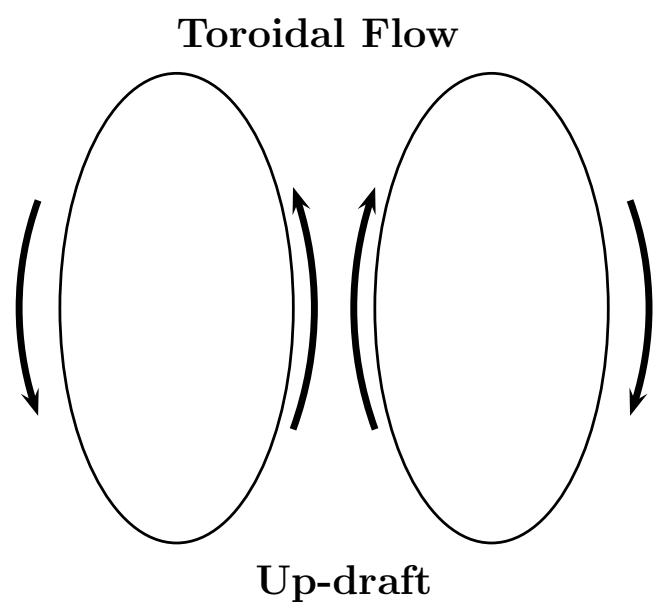

Fig. 8.- The Lorenz Model extended: convection in a sphere composed of a cell of toroidal shape. With no rotation to break the symmetry, the direction of the upflow vector is not constrained, and will be chaotically chosen.

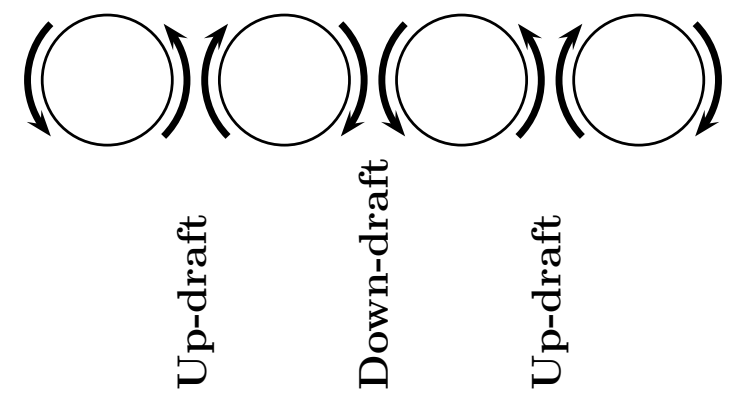

Fig. 9.- The Lorenz Model extended: Convection in a shell composed of cells. Notice the alternation of the sign of rotation. This may be thought of as a cross sectional view of infinitely long cylindrical rolls, or of a set of toroidal cells, with pairwise alternating vorticity (see Chandrasekhar (1961), §16). Each cell may exhibit independent fluctuations in time and space. tions. This approach is a simplified version of an elegant proposal by Lumley and collaborators (Holmes, Lumley, \& Berkooz 1996): empirical eigenfunctions are constructed by POD from simulations, introduced into the differential equations to derive a set of nonlinear ODE's for the amplitudes of each mode (a Galerkin projection), and this set of ODE's is solved to generate the evolution of the average properties of the turbulence. This is being explored (Meakin \& Arnett 2011) as a way to capture more fully the dynamics and multimodal behavior seen in the 3D simulations.

\subsection{Multiple Cells}

Suppose we envisage the convective region to be populated by cells, each of which is a separate Lorenz model representing the largest mode, a convective roll, in that cell. A special case is the convective core: the geometry is not that of a layer, but a sphere11, as is indicated in Figure 8. The flow has a toroidal structure. In this case the gravitational acceleration goes to zero at the origin (the center of mass of the star), but the velocity need not be zero there. If there is no net rotation, then the direction of the up-flow is undetermined, and will be chosen chaotically by the turbulent flow.

For a convective shell, we imagine that the layer is filled by cells, as shown schematically in Figure 9. For the oxygen burning shell, the inner and outer radii are about $4 \times 10^{8}$ and $8 \times 10^{8} \mathrm{~cm}$, respectively. The area of the spherical shell, evaluated at the midpoint in radius, is $4 \pi\left(6 \times 10^{8}\right)^{2}$, and the cell is taken to be roughly square, so that its area is $\ell^{2}=\left(4 \times 10^{8}\right)^{2}$. The ratio of shell area to cell area is about $9 \pi$, so we assume there are roughly 30 cells spread over the spheri-

\footnotetext{
${ }^{11}$ Woodward, Porter, \& Jacobs (2003) found such a "giant dipole" behavior in their 3D simulations of almost fully convective spheres.
} 
cal shell. In general, the number of cells in a shell will depend upon the geometry of the convection zone.

If the cells are not synchronous 12 , but act independently, the effect of the pulses will be smoothed when summed over the whole shell. However, the cells may interact constructively; the solution to this more complex problem remains open 13 . At issue are both the interactions between cells in a single convective region, and interactions between multiple convective regions associated with different burning shells.

The individual cells exhibit fluctuations not only in time, but also in space. Each cell represents a mode which is unstable, and destroys itself and reforms, usually somewhere else. Because the medium is fluid, the pattern of cells is much more dynamic and less regular than that of a crystalline solid, so the Fig. 9 should be interpreted as representing a snapshot of a system which fluctuates in both space and time. Hinode/SOT observations of the solar surface (Berger, et al. 2010) reveal such a highly dynamic, complex structure14.

\subsection{Irregular Variables}

Figure 10] explores an idea of Martin Schwarzschild (Schwarzschild 1975), who estimated the number of convective cells in the sun and in red giants and supergiants. He ar-

${ }^{12}$ See Alligood. Sauer \& Yorke (1996) for a discussion of synchronization of chaotic orbits.

13 Figure 23 in Meakin \& Arnett 2007b) suggests the complexity of the cell interaction within a single convective region. The original simulations were on a wedge, of $27^{\circ}$ in theta and in phi. Simulations with larger aspect ratio (larger angle wedges) do show a moderation of the total fluctuation, in qualitative agreement with the discussion above. Figure 2 in Meakin \& Arnett 2006) indicates the complexity of interaction between multiple burning regions even in $2 \mathrm{D}$.

${ }^{14}$ We thank Dr. A. Title for providing a copy of his spectacular movies of data and simulations. gued that only a modest number of cells $(\sim 12)$ would exist at any given time in a red giant or supergiant. To illustrate the point, we use the Lorenz model to approximate the behavior of a convective cell. We have estimated the convective flux for 12 cells at random phase, by adding 12 time sequences from an computation like Figure 4 (but extended to $\mathrm{t}=800$ ), starting at 12 randomly chosen times in this interval. This time sampling is intended to approximate a spatial ensemble average. Their flux, summed and normalized, is shown as a solid line, for a dimensionless time from 60 to 70 , which corresponds to roughly 20 pulses. The signal is noisy and looks "chaotic".

These fluctuating cells make up a convective region, and will couple to the normal modes of the star to cause both radial and non-radial pulsations. The amplitude of these pulsations will depend upon the overlap integrals between the normal modes and the cell motion, and the stellar damping. This suggests that the noisy behavior will be combined with the relatively cleaner periodicity of the normal modes, giving a power spectrum with a base like that shown in Figure 11, but with superimposed spikes corresponding to the excited normal modes. While turbulent convection alone is sufficient to cause luminosity fluctuations, it occurs in regions of high opacity and partial ionization, which also drive pulsation, so that composite behavior and multiple periods may be expected.

Joel Stebbins (pioneer of photoelectric astronomy) monitored the brightness of Betelgeuse ( $\alpha$ Orionis) from 1917 to 1931, and concluded that "there is no law or order in the rapid changes of Betelgeuse" (Goldberg 1984), which seems apt for Fig. 10 (the Lorenz strange attractor) as well. More modern observations (Kiss, Szabo, \& Bedding 2006) show a strong broad-band noise component in the photometric variability. The irregular fluctuations of the light curve are aperiodic, 


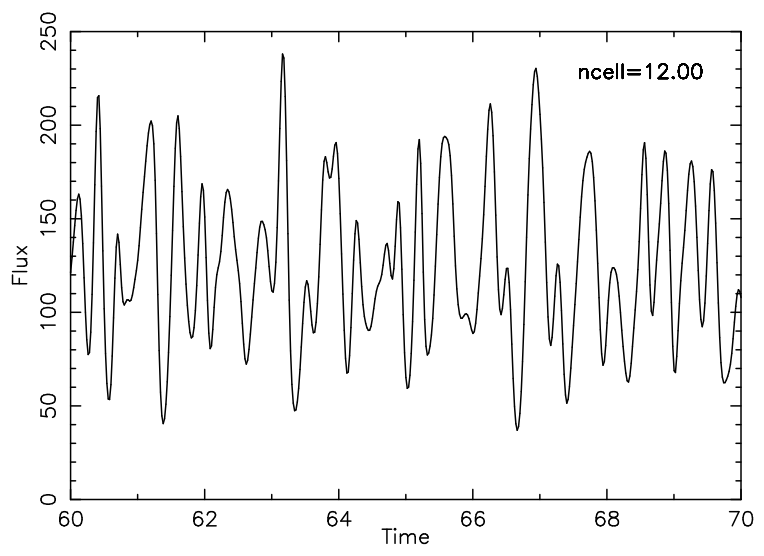

Fig. 10.- Fluctuations of Luminosity in Convective Layer of 12 Cells of Random Phase, for $\sigma=10, r=28$, and $b=8 / 3$. The dimensionless flux (luminosity) is shown for a convective layer with 12 visible Lorenz cells. The luminosity variations are large and seemingly chaotic, suggestive of irregular variables and Betelgeuse in particular (Kiss, Szabo, \& Bedding 2006).

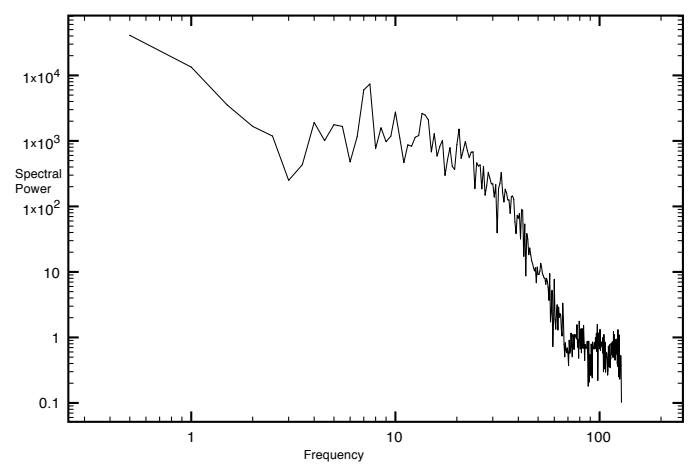

Fig. 11.- Power spectrum of the luminosity fluctuations implied by turbulence alone. There is no sharp peak, but a broad distribution of power, as would be expected from a chaotic source. Resonant interaction with normal modes of the star could add peaks to the spectrum, which would provide an observational constraint on interior structure, analogous to astro-seismology of solar-like stars. and resemble a series of outbursts. Direct 3D simulations of Betelgeuse (Chiavassa, et al. 2010) show the same complex behavior (and have the advantage that they predict the detailed spectral behavior as well). This should be no surprise; the 3D equations have embedded in them a strange attractor.

Increasing the number of cells reduces the level of fluctuation about the mean. Averaging over the 2 million granules of the sun gives a very stable luminosity, which would plot as a straight line in Fig. 10 (even 2000 random cells do this). However, the size of the cells at the bottom of the solar convection zone will be larger (hence fewer cells), and if chaotic might give a long term modulation to the solar luminosity. Full star simulations of the whole solar convective zone, with sufficient numerical resolution to give well developed turbulence, should shed light on this issue.

Application to turbulent stellar atmospheres requires surmounting two difficulties: (1) the flow is no longer low-Mach number (see $\S 2.2$ ), and (2) the ionization zone causes dramatic changes in opacity (assumed constant in the Lorenz model). Fortunately 3D atmospheres exist, and analysis such as we have done on stellar interior convection simulations is feasible.

While this paper was in preparation, Stothers (2010) re-examined the idea that giant convective cell turnover is the explanation of the long secondary period observed in semiregular red variable stars (Stothers \& Leung 1971), including Betelgeuse and Antares. Stothers used MLT to derive a velocity scale for the overturn, also relying on general features 15 of simulations of Chan \& Sofia (1996); see $\S 3$ in Stothers (2010). This theory appears to work directly as a complement to the discussion above. The use of a Lorenz

15 Arnett, Meakin, \& Young (2009) discuss and contrast these and other simulations. 
model for the giant cells already implies real dynamics. The strange attractor necessarily provides variability in luminosity, with its own quasi-period (Wood. Olivier \& Kawaler 2004) and velocity scale (see $\S 4$ in Stothers (2010)). Several giant cells 16 are at work, each with a quasi-period of order of the transit time, and therefore similar to the estimate of Stothers, and the observations. The larger convective velocities needed are simply a necessary consequence of the dynamics implied by the convective luminosity (Arnett. Meakin, \& Young 2010).

The introduction of turbulence as an active agent in the discussion of stellar variability (e.g., Stothers (2010); Wood, Olivier \& Kawaler (2004)) seems timely. An interesting improvement would be to use POD empirical eigenvalues from simulations (e.g., Chiavassa, et al. (2010), $§ 5.1$ above), and develop a low order dynamical model to explore long duration behavior.

\section{Summary}

We have identified a major new feature of stellar physics: chaotic behavior due to turbulent fluctuations in stellar convection, and corresponding luminosity fluctuations. While the simulations upon which the analysis was based were fully compressible, the theory uses the approximation of sub-sonic flow. Both numerically and analytically, a strange attractor like that of Lorenz (1963) seems to appear naturally in stellar convection.

As a first approximation to more rigorous analysis, we have applied the Lorenz model to kinetic energy fluctuations in the oxygen burning shell, to the turbulent energy cascade, and to fluctuations in luminosity in irregular

${ }^{16}$ This idea can be tested observationally and numerically; the structure of the convection region will constrain the number of cells, which may be compared with the amplitude of luminosity fluctuations. variables.

Figure 11 shows a comparison of the turbulent kinetic energy fluctuations in 3D simulations of turbulent flow and in the Lorenz model. No parameters were adjusted to give a fit. Additional modes, appropriate for turbulent flow, would improve the comparison further.

This suggests a new, inherently nonlinear mechanism for variability in stars, the $\tau$-mechanism, which is caused by luminosity fluctuations directly associated with turbulent convective cells. Because the mechanism is nonlinear, it is not captured by linear stability analysis, which is a mainstay of variable star theory. Such luminosity fluctuations may have been observed already in the broad-band noise seen in Betelgeuse (alpha Orionis Goldberg 1984; Kiss, Szabo, \& Bedding 2006), and in the long secondary periods in pulsating AGB stars (Wood, Olivier \& Kawaler 2004; Stothers 2010), and are expected to be observable in principle in all stars with extensive surface convection zones, including those with "solar-like" variability. This mechanism is probably the cause of the strongly driven pulsations found by Woodward. Porter, \& Jacobs (2003) in their "red giant" model; the development of those large pulsations was a clue which may now be more fully understood.

Such fluctuations provide a source of perturbations for instabilities, and may induce mixing not presently accounted for in stellar evolutionary calculations. The fluctuations in convective velocity are comparable to average values. If these fluctuations couple to nuclear burning, as for example in cases of degenerate ignition, shell flashes, or later stages of oxygen and silicon burning 17 , outbursts may develop.

\footnotetext{
${ }^{17}$ For new developments since this paper was submitted, see Arnett \& Meakin (2011b), where it is suggested that core collapse progenitors are dynamically active
} 
This work was supported in part by NSF Grant 0708871 and NASA Grant NNX08AH19G at the University of Arizona, and by the CLEAR sub-contract from University of Michigan. We wish to thank Fr. J. Fuñes (Specalo Vaticano), Prof. R. Ruffini (ICRAnet), and Prof. J. Lattanzio (Monash), P. Wood (Australian National University), and the Aspen Center for Physics for their hospitality, Prof F. Timmes and S. Starrfield for discussions, R. Stothers for helpful email, and the second anonymous referee for constructive comments.

prior to collapse. 


\section{A. APPENDIX: Physical Basis of the Lorenz Model}

Mass conservation. Conservation of mass is enforced by use of a stream function (Landau \& Lifshitz (1959), §9); the simplest solution is a two dimensional, cylindrical "roll" (Chandrasekhar (1961), p. 44). The flow is assumed to be subsonic.

Momentum conservation. The Navier-Stokes equation may be written as

$$
D \mathbf{u} / D t=\nu \nabla^{2} \mathbf{u}-\mathbf{g} \Delta \rho / \rho,
$$

in the low Mach number limit; the last term is the buoyant acceleration. The flow executes a circle of radius $\ell / 2$; see Figure 3. The density fluctuation is related to the temperature fluctuation by $\Delta \rho / \rho_{0}=\beta_{T}\left(T-T_{0}\right) / T_{0}$, where $\beta_{T}=-(\partial \ln \rho / \partial \ln T)_{P}$.

We separate the variable $\mathbf{u}$ by considering the flow to be a mass flux which is constant around the ring at any given moment, represented by an average speed $u$ which is a function of time only 18 . The hydrostatic background in the convective region has an entropy $S$ which is constant. Then,

$$
\frac{1}{\rho} \frac{d P}{d r}=\frac{d W}{d r}=-g
$$

where $W=E+P V$ is the enthalpy per unit mass, and $W=C_{P} T$, and $C_{P}=\frac{\gamma}{\gamma-1} \mathcal{R} Y$. Now $\int g d r=g \Delta r$ if $g$ is constant. Lorenz assumed a linear temperature decrease with height, which corresponds to constant gravitational acceleration $g$. Using a height $z=(\ell / 2) \cos \phi$,

$$
W(\phi)=W(0)+g(\ell / 2) \cos \phi
$$

or

$$
T(\phi)=T_{0}+\frac{g(\ell / 2)}{C_{P}} \cos \phi
$$

We denote $T_{1}=g(\ell / 2) / C_{P}$, so

$$
T_{E}=T_{0}+T_{1} \cos \phi
$$

which corresponds to the environmental temperature. For $T_{1}>g / C_{P}$ the system is convectively unstable, while for $T_{1}<T_{0}$, the background temperature can never be negative. We represent the temperature by

$$
T=T_{0}+T_{2} \cos \phi+T_{3} \sin \phi
$$

Viscous damping. The viscous damping term may be approximated by $\nu \nabla^{\mathbf{2}} \mathbf{u} \rightarrow-\Gamma u=$ $-\nu(2 / \ell)^{2} u$. Here the constant $\Gamma$ is the inverse of the viscous dissipation time scale $\tau_{v i s}$.

The buoyant acceleration in the vertical direction is

$$
B_{z}=-\mathbf{g} \cdot(\delta \rho / \rho) \rightarrow g \beta_{T}\left(2 T_{3} / T_{0}\right) \sin ^{2} \phi .
$$

Only a temperature (buoyancy) difference in the horizontal direction $(\phi=\pi / 2)$ gives a net torque to turn the convective roll.

18 Variations in density due to change in hydrostatic stratification could be included as well as the implied changes in velocity and cross-sectional area; see Tritton (1988), p. 188-196). 
With damping due to a linear viscosity term,

$$
d u / d t=-\Gamma u+\frac{g \beta_{T}}{T_{0}}\left(T-T_{E}\right) \sin \phi .
$$

Integrating Eq. A8 over a complete cycle in $0 \leq \phi \leq 2 \pi$, gives 19

$$
d u / d t=-\Gamma u+\left[\frac{g \beta_{T}}{2 T_{0}}\right] T_{3}
$$

The temperature terms in $\cos \phi$ integrate to zero because of the $\sin \phi$ factor in the buoyancy term. Thus we have just two terms in Eq. A9, a sink from the viscous damping and a source from buoyancy.

In the turbulent case, we might identify this rate of dissipation as an integral over the turbulent region, so it is transformed into a global quantity, $\varepsilon_{K} / \rho=u^{3} / \ell$. This is the deceleration times the velocity, giving a deceleration of $-u|u| / \ell$, so that $\nu \nabla^{\mathbf{2}} \mathbf{u} \rightarrow-u|u| / \ell$. The absolute value $|u|$ is used here because the characteristic time is $\tau_{v i s}=\ell /|u|$, and the deceleration is thus $-u / \tau_{v i s}$. The length scale is the depth of the convective shell (i.e., $\ell$ ). The convective speed $u$ is constant in space, so we may use a nonlinear damping term, as implied by Kolmogorov (1941),

$$
d u / d t=-u|u| / \ell+\left[\frac{g \beta_{T}}{2 T_{0}}\right] T_{3}
$$

Energy conservation. At constant pressure, the first law of thermodynamics simplifies to

$$
d W / d t=C_{P} d T / d t=\epsilon-\frac{1}{\rho} \nabla \cdot \mathbf{F}+\frac{\varepsilon_{K}}{\rho},
$$

where $\epsilon$ is the net heating-cooling rate from nuclear burning and neutrino emission, $C_{P}$ the specific heat at constant pressure, $T$ the temperature, $\rho$ the mass (nucleon) density, $\mathbf{F}$ the flux of radiative energy, and $\varepsilon_{K}$ the volumetric heating by turbulence. Ignoring $\epsilon$ and $\varepsilon_{K}$,

$$
\partial T / \partial t+\mathbf{u} \cdot \nabla \mathbf{T}=-\left(\frac{1}{\rho} \nabla \cdot \mathbf{F}\right) / C_{P}
$$

The divergence of flux of radiative luminosity is

$$
\frac{1}{\rho} \nabla \cdot \mathbf{F}=-\frac{1}{\rho} \nabla \cdot\left[-(c / 3 \rho \kappa) \nabla a T^{4}\right],
$$

so that

$$
\partial T / \partial t+\mathbf{u} \cdot \nabla T=\nu_{T} \nabla^{2} T
$$

where $\nu_{T}=\left(4 a c T^{3} / 3 \rho \kappa\right) /\left(\rho C_{P}\right)$ is the thermal conductivity for radiative diffusion, for constant $\kappa$ and $C_{P}$.

The background temperature $T_{E}$ is chosen such that $\nabla^{2} T_{E}=0$. The radiative diffusion term is

$$
\nu_{T} \nabla^{2} T \approx K\left(T_{E}-T\right),
$$

\footnotetext{
${ }^{19}$ The two terms constant in space $(\phi)$ generate a factor of $2 \pi$ while the integral over $\sin ^{2} \phi$ gives a factor of $\pi$.
} 
where $K\left(T_{E}-T\right)$ is the extra radiative diffusion due to the deviation of temperature from $T_{E}$, and $K=\nu_{T}(2 / \ell)^{2}$ is the inverse of the radiation diffusion time scale $\tau_{\text {rad }}$.

The classical Lorenz equations. Following Lorenz (1963), we correct for an aspect ratio $a$ by a factor $b=4 /\left(1+a^{2}\right)$ which deals with the excess in vertical over horizontal heat conduction $(b K$ versus $K$ ). Substituting for $T$ and $T_{E}$ in Equation A14, we have

$$
\begin{array}{r}
\frac{d T_{2}}{d t} \cos \phi+\frac{d T_{3}}{d t} \sin \phi \\
-\frac{2 u T_{2}}{\ell} \sin \phi+\frac{2 u T_{3}}{\ell} \cos \phi \\
=b K\left(T_{1}-T_{2}\right) \cos \phi-K T_{3} \sin \phi .
\end{array}
$$

Coefficients of orthogonal functions must separately sum to zero, so

$$
\begin{aligned}
& d T_{3} / d t-2 u T_{2} / \ell=-K T_{3} \\
& d T_{2} / d t+2 u T_{3} / \ell=b K\left(T_{1}-T_{2}\right) .
\end{aligned}
$$

We introduce a potential temperature

$$
T_{4}=T_{1}-T_{2}
$$

and eliminate $T_{2}$. If $T_{1}=g \ell / 2 C_{P}$ is independent of time,

$$
\begin{array}{ccc}
d T_{3} / d t= & -K T_{3}+2 u T_{1} / \ell-2 u T_{4} / \ell \\
d T_{4} / d t= & -b K T_{4}+2 u T_{3} / \ell .
\end{array}
$$

We define dimensionless variables

$$
\begin{aligned}
\tau & =t K \\
X & =u(2 / \ell K) \\
Y & =T_{3}\left(g \beta_{T} / \ell \Gamma K T_{0}\right) \\
Z & =T_{4}\left(g \beta_{T} / \ell \Gamma K T_{0}\right)
\end{aligned}
$$

and use Equations A9, A20, and A21 to get the Lorenz equations in their usual form: Equations 12, 13, and 14.

$$
\begin{array}{rlrl}
d X / d \tau & = & & -\sigma X+\sigma Y \\
d Y / d \tau & = & -X Z+r X-Y \\
d Z / d \tau & = & X Y-b Z,
\end{array}
$$

where $\tau$ is a time in thermal diffusion units, $\sigma=\Gamma / K$ is the effective Prandtl number, and $r=$ $\left(g \beta_{T} T_{1} / \ell \Gamma K T_{0}\right)=\left(g \beta_{T} T_{1} / \ell \Gamma^{2} T_{0}\right) \sigma$ is the ratio of the Rayleigh number to its critical value for onset of convection. The Prandtl number is the ratio of coefficients of the viscous dissipation term to the thermal mixing term, $\sigma=\nu / \nu_{T}=\Gamma / K=\tau_{\text {rad }} / \tau_{v i s}$, where $\tau_{v i s}=1 / \Gamma$ is the viscous damping time. 


\section{B. APPENDIX: What Should The Prandtl Number Be?}

The original Lorenz parameters appear to be a fair choice to represent the flow in 3D simulations (see Figure 1). This is consistent with an effective Prandtl number for the numerical simulation of $\sigma_{t} \approx 10$; however, the severe reduction in degrees of freedom from the simulations to the Lorenz model warns against taking the numerical value too literally. We note for comparison that water has $\sigma \approx 6$ and air has $\sigma \approx 0.7$. Apparently Lorenz felt he was lucky (Lorenz (1993), p. 137); he took a suggested value $(\sigma=10)$ which gave chaotic behavior instead of a lower value, actually appropriate for air, for which his equations give stable rolls. As a mathematical example this quantitative difference in the Prandtl number is not significant, but for physical applications, it is.

\section{B.1. The Microscopic Value}

Using the ratio of microscopic thermal diffusion to viscous time scale, Hansen \& Kawaler (1994) (page 178 and 185) suggest that $\sigma \approx 10^{-8}$. Lorenz (1963) finds the critical value for $r$ for instability of steady convection to be $r_{c}=\sigma(\sigma+b+3) /(\sigma-b-1)$. For $\sigma<b+1=2.66 \overline{6}$, steady convection is always stable, so that the Hansen \& Kawaler (1994) value would never give turbulence. If $\sigma>b+1$, steady convection is unstable for sufficiently large Rayleigh numbers. This precise value for instability is a characteristic of the canonical Lorenz model, and is affected by the particular choice of dissipation function (see Appendix A).

\section{B.2. The Simulation Value}

In the numerical simulations, the effective Prandtl number is dominated by the turbulent cascade, which gives mixing of both momentum and heat at a rate determined by the largest eddy size. Thus, in numerical simulations, the turbulent flow defines its own effective value of this parameter $\sigma \rightarrow \sigma_{t}$, and whatever value $\sigma_{t}$ has, it is clearly above the threshold for instability for the system we have numerically simulated. Numerical simulations and experiment suggest, for developed turbulence at high Reynolds numbers, $\sigma_{t} \approx 0.7$, a value typical of many common gases.

We note that in the "Reynolds analogy" (Monin \& Yaglom (1971), p. 341), Osborne Reynolds argued that the mechanisms for transport of heat and momentum were essentially the same in a turbulent medium, so that the effective turbulent Prandtl number should be of order unity $\left(\sigma_{t} \approx 1\right)$.

\section{B.3. A Cascade Estimate}

Suppose we think of the Prandtl number as the relative strength of the process which makes the velocity field isotropic to that which converts the kinetic energy into heat. These occur at different ends of the turbulent cascade. To better understand what this might mean, consider $\sigma=$ (time to change direction)/(time to heat). We approximate the time to change direction by the time to halve the kinetic energy, $\left(\ell / 2 v_{r m s}\right)$. At any length scale $\lambda$ the turbulent cascade has a transfer rate for kinetic energy of $v_{\lambda}^{3} / \lambda=v^{3} / \ell$. This implies a time spent at each length scale of $\tau_{\lambda}=\lambda / v_{\lambda}$. If each level in the cascade is smaller on average in length scale by a factor $\lambda(n+1) / \lambda(n)=f$, the total time for the cascade is $(\ell / v)\left(1+f^{2 / 3}+\cdots\right)=(\ell / v) /\left(1-f^{2 / 3}\right)$, where the geometric series has been used for the summation (see also Frisch (1995), §7.8). This gives an effective Prandtl number $\sigma_{t} \approx 2 /\left(1-f^{2 / 3}\right)$. Some representative values: for $f=1 / e$, this gives $\sigma \approx 4.1$, for $f=1 / 2$ this gives $\sigma \approx 5$, and $f=1 / \sqrt{2}$ gives $\sigma \approx 10$. This argument may tend to overestimate the Prandtl number, so that $\sigma_{t}$ would be smaller than the historical value chosen by Lorenz $(\sigma=10)$. 


\section{B.4. Renormalization Group}

The separation in size of the large scale eddies with those at the dissipation scale suggests that this coupling might be approximated in some ingenious way. The microscopic viscosity might be considered a "bare" value, to be renormalized to a "dressed" value, in analogy to the field-theoretic treatment of interacting particles. Kadanoff (1966) proposed the idea of reducing the size of a system a step at a time by grouping neighboring entities (in this case molecules) and treating each group as a single interaction. Wilson (1970) has successfully implemented the general idea of coarse-graining, or "weeding out the small scales". These general ideas may be applied to the turbulent cascade. Yakhot \& Orszag (1986) use renormalization-group (RG) analysis of turbulence with some success, and in particular, estimate the effective Prandtl number for turbulent flow to be $\sigma_{t}=0.7179$; see also Kraichnan (1987).

However there is some debate: in their review, Smith \& Woodruff (1998) warn "...the RG method ... leads to suggestive results when applied to turbulence... However, its application to turbulence cannot yet be called a major success, owing to the uncontrolled approximations currently required to implement it." A similar sentiment is found in McComb (2004), p. 290.

\section{B.5. A Perspective}

We have captured the behavior of the pulses in the simulations, by a Lorenz model using the parameters that Lorenz used. In this sense, these values are relevant to our problem, although it is unclear from first principles what the precise value of the effective turbulent Prandtl number should be. The form for dissipation that Lorenz used is not the same as implied by Kolmogorov, so that the threshold for instability changes (Arnett \& Meakin 2011c). We may interpret the Prandtl number $\sigma$ and the Rayleigh number $r$ in terms of a renormalization in which the existence of turbulence implies effective Prandtl and Rayleigh numbers for the convective cell. The actual values of the microscopic viscosity and thermal conductivity have little feedback on the behavior of the largest eddies; see Section 2.1. Magnetic fields in real stars may affect the effective Prandtl number further, which in the classical Lorenz model affects in turn the development of instability in the convective roll.

For problems which are insensitive to the details of the small scale flow, the values of the microscopic Prandtl do not matter; the turbulent system bootstraps to an effective dissipation for which the rules of Kolmogorov hold. This approximation applies to hydrostatic (and mildly dynamic) stellar evolution, in which the burning times are long compared to sound travel times. For these problems, ILES is appropriate. There are notable exceptions, such as a flame front in a medium of unmixed fuel and ash (SNIa progenitor models), for which the small scales are important, and direct numerical simulations (DNS) of the small scales is necessary.

Finally, we recall how the Lorenz model was constructed: a low order mode was chosen, a Rayleigh number was chosen just above the onset of instability for that mode, and a Prandtl number was chosen which gave interesting behavior. While different parameter choices are mathematically interesting (Sparrow 1982), their physical relevance must be re-evaluated. The Lorenz equations are a spartan subset of the fluid equations which contain the germ of chaos; it is probably better to use them as a guide rather than a gospel. 


\section{REFERENCES}

Alligood, K. T., Sauer, T. D., \& Yorke, J. A., 1996, Chaos: An Introduction to Dynamical Systems, Springer, Berlin

Arnett, D., 1972, ApJ, 176, 681

Arnett, D., 1996, Supernovae and Nucleosynthesis, Princeton University Press, Princeton NJ

Arnett, W. D., Meakin, C., \& Young, P. A., 2009, ApJ, 690, 1715

Arnett, W. D., Meakin, C., \& Young, P. A., 2010, ApJ, 710, 1619

Arnett, D., \& Meakin, C., 2011b, submitted.

Arnett, D., \& Meakin, C., 2011c ApJ, submitted

Berger, T. E., Slater, G., Hurlburt, N., Shine, R., Tarbel, T., Title, A., Lites, B. W., Okamoto, R. J., Ichimoto, K., Katsukawa, Y., Magara, T., Suematsu, Y., Shimizu, T., 2010, ApJ, 716, 1288

Boris, J., 2007, in Implicit Large Eddy Simulations, ed. F. F. Grinstein, L. G. Margolin, \& W. J. Rider, Cambridge University Press, p. 9

Böhm-Vitense, E., 1958, ZAp, 46, 108

Canuto, V. M. \& Mazzitelli, I., 1991, ApJ, 370,295

Canuto, V. M., Goldman, I., \& Mazzitelli, I., ApJ, 473, 550

Chandrasekhar, S. 1961, Hydrodynamic and Hydromagnetic Instability, Oxford University Press, London

Chan, K. L., \& Sofia, S. 1996, ApJ, 466, 372

Chiavassa, A., Haubois, X., Young, J. S., Plez, B., Josselin, E., Perrin, G., \& Freytag, B., 2010, A\&A, in press
Cvitanović, P., Universality in Chaos, Adam Hilger, Bristol and New York

Davidson, P. A., 2004, Turbulence, Oxford University Press, Oxford

Dutton, J. A., 1986, The Ceaseless Wind, Dover Publications, Inc., Mineola, NY

Frisch, U., 1995, Turbulence, Cambridge University Press, Cambridge

Gleick, J., 1987, Chaos: Making a New Science, Penguin Books, New York

Goldberg, L., 1984, PASP, 96, 366

Hansen, C. J., \& Kawaler, S. D., 1994, Stellar Interiors, Springer-Verlag

Holmes, P., Lumley, J. L., \& Berkooz, G., 1996, Turbulence, Coherent Structures, Dynamical Systems, and Symmetry, Cambridge University Press

Kadanoff, L. G., 1966, Physics, 2, 263

Kiss, L. L., Szabo, Gy. M., \& Bedding, T. R., 2006, MNRAS, 372, 1721

Kolmogorov, A. N., 1941, Dokl. Akad. Nauk SSSR, 30, 299

Kolmogorov, A. N.,1962, J. Fluid Mech., 13, 82

Kraichnan, R. H., 1987, Phys. Fluids, 30, 2400

Landau, L. D., 1944, C. R. (Dokl.) Acad. Sci. URSS 44, 311

Landau, L. D. \& Lifshitz, E. M. 1959, Fluid Mechanics, Pergamon Press, London

Ledoux, P., 1941, ApJ, 94, 537

Libchaber, A., \& Mauer, J., 1982, in Nonlinear Phenomena at Phase Transitions and Instabilities, ed. T. Riste, 259-86, Plenum Publ. Corp. 
Lorenz, E. N., 1963, Journal of Atmospheric Sciences, 20, 130

Lorenz, E. N., 1995, The Essence of Chaos, University of Washington Press, Seattle

McComb, W. D., 2004, Renormalization Group, A Guide for Beginners, Clarendon Press, Oxford

Meakin, C., \& Arnett, D., 2006, ApJ, 637, 53

Meakin, C., \& Arnett, D., 2007a, ApJ, 665, 690

Meakin, C., \& Arnett, D., 2007b, ApJ, 667, 448

Meakin, C., \& Arnett, D., 2010, Ap\&SS, 328, 221

Meakin, C., \& Arnett, D., 2011 ApJ, submitted

Monin, A. S. \& Yaglom, A. M., 1971, Statistical Fluid Mechanics: Mechanics of Turbulence, vol. 1, Dover Publications, Mineola NY

Nordlund, A., Stein, R., \& Asplund, M., 2009, http://ww.livingreviews.org/lrsp-2009-2

Obukhov, A. M., 1962, J. Fluid Mech., 13, 77

Poincaré, H., 1893, Les Methodes Nouvelles de la Mécanique Céleste, Gauthiers-Vilar, Paris

Ruelle, D., \& Takens, F., 1971, Commun. Math. Phys. 20, 167

Schwarzschild, M., 1975, ApJ, 195, 137

Shannon, C., 1948, Bell System Technical Journal, 27, 379

Sparrow, C. 1982, The Lorenz Equations: Bifurcations, Chaos, and Strange Attractors, Springer-Verlag, Berlin
Smith, L. M., \& Woodruff, S. L., 1998, Ann. Rev. Fluid Mech., 30, 275

Stothers, R. B., \& Leung, K.-C., A\&A, 10, 290

Stothers, R. B., 2010, ApJ, 725, 1170

Sytine, I., Porter, D., Woodward, P., Hodson, S. W., \& Winkler, K-H., 2000, J. Chem. Phys., 158, 225

Thompson, J. M. T. \& Stewart, H. B., 1986, Nonlinear Dynamics and Chaos, John Wiley and Sons, New York

Tritton, D. J., Physical Fluid Dynamics, 2nd ed., Oxford University Press, Oxford UK

Unno, W., Osaki, Y., Ando, H., Saio, H., \& Shibahashi, H., 1989, Nonradial Oscillations of Stars, 2nd. ed., University of Tokyo Press, Tokyo

von Neumann, J., 1948, in Collected Works, Volume VI, 1963, Pergamon Press, Oxford, p. $467-9$

Wilson, K. G., 1970, Phys. Rev. D2, 1438

Woodward, P. R., Porter, D. H., \& Jacobs, M., 2000, in 3D Stellar Evolution, ed. S. Turcotte, S. C. Keller, \& R. M. Cavallo, ASP Conference Series 293, p. 45

Wood, P. R., Olivier, E. A., and Kawaler, S. D., 2004, ApJ, 604, 800

Woodward, P. R., Porter, D., Anderson, S., \& Fuchs, T., 2006, in Numerical Modeling of Space Plasma Flows, ed. N. V. Pogorelov \& G. P. Zank, Astron. Soc. Pacific Conf. Series, 359, 97

Woodward, P. R., 2007, in Implicit Large Eddy Simulantions, ed. F. F. Grinstein, L. G. Margolin, \& W. J. Rider, Cambridge University Press, p. 130 
Woosley, S. E., Heger, A., \& Weaver, T. A., 2002, Rev. Mod. Phys., 74, 1015

Yakhot, V., \& Orszag, S. A., 1986,

Phys. Rev. Lett.57, 1722

This 2-column preprint was prepared with the AAS LATEX macros v5.2. 